\title{
PRODUÇÃO TECNOLÓGICA BRASILEIRA NA ÁREA DE ENFERMAGEM: avanços e desafios
}

\author{
M icheline Henrique A raujo da L uz KOE RICH ${ }^{a}$, Raquel H el oisa G uedes VIEIRA ${ }^{b}$, D aniela E da SI LVA ${ }^{c}$, \\ Alacoque Lorenzini ERD M AN N d, Betina H orner Shlindwein M E IRE LLES
}

\section{RESUMO}

Este artigo objetiva analisar as patentes registradas na área de enfermagem já que as mesmas podem configurar como um dos indicadores do seu desenvolvimento. A presenta e discute as produções tecnológicas em âmbito nacional, rastreadas pelo termo "enfer magem", patenteadas no período de 1990-2009. T rata-se de uma pesquisa documental, retrospectiva tendo como fonte o banco de dados da Base de Pedidos de Patentes (BRASPAT), do Instituto N acional da Propriedade Industrial (I N PI ). A s infor mações obtidas são discutidas no tocante a apropriação das tecnologias, o incentivo para desenvolvê-las e registrá-las como fonte de conhecimento da ár ea de enfermagem, com vistas a prática do cuidado. São crescentes as produções de tecnologias leves e leve-duras na enfermagem, todavia, estas não são registradas e patenteadas. 0 avanço tecnológico na enfer magem é emergente e carece de políticas para seu incremento.

D escritores: Pesquisa em enfermagem. Tecnologia biomédica. Patentes.

\section{RESUMEN}

E ste artículo tiene como objetivo analizar las patentes registradas en la área de enfermería ya quelas mismas puedeconfigurarse como uno de los indicadores de su desar rollo. P resenta y discute acerca de la producción tecnológica nacional valiéndose de la palabra enfermería patentados entre 1990-2009. E sta es una investigación documental, retrospectiva, teniendo como fuente de datos e Instituto N acional de P roductos Industriales (IN P I). L as informaciones obtenidas son discutidas en términos de la apropiación de las tecnologías, los incentivos para desar rollarlas y registrarlas como fuente de conocimi entos de enfer mería, con la mirada en las prácticas de cuidado. E s creciente el aumento de la producción de tecnología blanda y suave duro en la enfermería, sin embargo, no son registradas ni patentadas. E I avance tecnológico en la enfermería es emergente y necesitamos políticas para promover la mejora de la misma.

Descriptores: Investigación en enfer mería. Tecnología biomédica. Patentes. T ítulo: $L$ a producción tecnológica brasileña en enfermería: avances y desafíos.

\section{ABST RACT}

This article aims to analyze the patents register ed in the nursing area, since these patents may be used as an indicator of the technological development in thearea. It presents and discusses national technological productions, tracked through the "nursing" keyw ord, patented in the period from 1990-2009. T his is a retrospective documental research, using, as a source, data from the $\mathrm{N}$ ational Industrial P roperty Institute (IN P I). T he information gathered is discussed in relation to the appropriation of the technologies, the incentive to develop them and register them as a source of knowledge in the nursing field, aiming the practice of care $\mathrm{L}$ ight and light hard technology productions areincreasing in the nursing field. $\mathrm{H}$ ow ever, these are not registered and patented. The technological advance in the nursing field is emergent and needs policies for its development.

D escriptors: N ursing research. Biomedical technology. Patents.

T itle: Brazilian technological output in the area of nursing: advances and challenges.

\footnotetext{
a M estre em Ciências do M ovimento Humano, A luna do Programa de Pós-G raduação em Enfermagem (PEN) na U niversidade Federal de Santa Catarina (U F SC), F lorianópolis, Santa Catarina, Brasil.

${ }^{b}$ E nfer meira, M estranda do M estrado Profissional em G estão do Cuidado em E nfer magem (U F SC), E specialista do A mbul atório de Pediatria do Hospital U niversitário (U F SC), F lorianópolis, Santa Catarina, Brasil.

M estre em Saúde Coletiva, Professora T itular do D epartamento de Enfermagem da U niversidade de Ciências da Saúde de Alagoas (UNCISAL), M aceió, Alagoas, Brasil.

d D outora em Filosofia da Enfermagem, Professora T itular do Departamento de Enfermagem e do PEN da UFSC, Pesquisadora 1 A do Conselho Nacional de Desenvolvimento Científico e Tecnológico (CN Pq), Coordenadora da Área da Enfermagem na Coordenação de A perfeiçoamento de Pessoal de Nível Superior (CAPES), F lorianópolis, Santa Catarina, Brasil.

D outora em Filosofia da Enfermagem, Professora Adjunta do Departamento de Enfermagem e do PEN da UFSC, Florianópolis, Santa Catarina, Brasil.
} 


\section{INT RODUÇÃO}

0 homem, desde seus primórdios, sempre se interessou por novidades, contudo o caráter técnico-científico das invenções e inovações somente veio a emergir no período compreendido entre a Revolução Industrial e a Segunda Grande Guerra, em um movimento que levou ao surgimento e aperfeiçoamento de teorias, métodos e processos científicos característicos de uma tecnologia(1).

A partir deste período, o mundo e a humanidade vêm sofrendo mudanças importantes no seu ser e fazer. Todas essas transformações estiveram ou estão de algum modo, relacionadas à criação, incorporação, aperfeiçoamento e manipulação de novas tecnologias em uma ação contínua e intensamente crescente de novas descobertas ${ }^{(2,3)}$.

D entro desta concepção, a tecnologia transcende ao objeto/ máquina, pois inclui também os conhecimentos e métodos usados na produção de bens e serviços, além daqueles relacionados a processos de organização(4). Sua classificação tradicionalmente tem sido expressa pelos termos de "dura"; "leve-dura" e "leve", sendo que tecnologias duras seriam aquelas relacionadas aos objetos e maquinários; as leves-duras seriam compostas pelos saberes estruturados e as leves por processos que influenciam as relações entre os indivíduos ${ }^{(1,5)}$. V ista sob este prisma, pensar tecnologia é pensar num leque extremamente diversificado de possibilidades e desafios.

Inventar é descobrir novos produtos ou processos, já inovar é introduzir e implementar no mercado ou na prática social esse produto novo ou significativamente aperfeiçoado tendo por base o conhecimento disponível em determinado momento da história ${ }^{(3,4,6)}$.

Os enfermeiros estão expostos diariamente às novas tecnologias, quer em sua vida pessoal, quer em sua atividade profissional, e, quanto mais rápido apreendê-las e dominá-las, colocando-as a serviço do ser humano, mais avanços terão em sua profissão. Sob este prisma, é importante que as pesquisas científicas e tecnológicas sejam conhecidas e consumidas pelo profissional da enfermagem a fim de permitir uma melhor qualidade do cuidado.

$M$ as, além de consumir novas tecnologias, acredita-se que 0 enfermeiro deva transformar sua prática diária criando, gerando inovações, esti- mulando para que sejam testadas e sistematizadas através de pesquisas científicas. $N$ a década de 1990 , um projeto desenvolvido pela U niversidade Federal de Santa Catarina (U F SC) ${ }^{(7)}$, levou ao desenvolvimento de 33 produtos, demonstrando o potencial da enfermagem na produção de tecnologias. No entanto, apenas nove desses inventos foram patenteados, refletindo a necessidade de introduzir a concepção de preservação de autoria como sinal de domínio e reconhecimento do saber na profissão.

No Brasil, a instituição que concede 0 direito de patente, ou seja, que permite a exploração da invenção/ inovação é o Instituto N acional de Propriedade Industrial (INPI), uma autarquia vinculada ao M inistério do Desenvolvimento, Indústria e Comércio $E x t e r i o{ }^{(4)}$. A introdução e a incorporação de uma nova tecnol ogia em saúde, por sua vez, são reguladas pela $A$ gência $\mathrm{N}$ acional de $V$ igilância Sanitária (ANVISA) e, para que esta venha a fazer parte dos procedimentos do Sistema Ú nico de Saúde (SU S), sua regulamentação é realizada pela Secretaria deA tenção à Saúde(SA S), órgão do M inistério da Saúde (M S) que se encarrega de distribuir os recursos para o três níveis de atenção em saúde $e^{(2)}$.

Além das questões legais e jurídicas, alguns aspectos dificultam a introdução de uma nova tecnologia em nosso meio, como por exemplo, aqueles relacionados à formação e reciclagem de recursos humanos para o uso adequado das mesmas, a atual ização constante de instrumentos para regular/ certificar novos produtos e a exigência constante de investimentos em infra-estrutura física adequada.

Outro aspecto que não pode ser esquecido é o impacto que as novas tecnologias exercem diretamente na qualidade do serviço prestado, alimentando a geração, não só de novas invenções/ inovações, mas também, de novas necessidades para o setor ${ }^{(6)}$.

Ressalte-se ainda a carência no desenvolvimento de novas tecnologias nativas, obrigando importações frequentes que ocasionam aumento nos custos, e possíveis inadequações às necessidades específicas e particulares do contexto onde serão inseridas ${ }^{(7)}$.

A partir dos anos 1950, a Enfermagem desenvolveu e aperfeiçoou tecnologias relacionadas ao cuidado de modo empírico, improvisado e de baixo custo, impulsionada pelas deficiências geradas na prática diária e urgência de resposta imediata para suprir as necessidades do cliente. M uitas das tecno- 
logias assim desenvolvidas não estavam embasadas em pressupostos científicos, evidenciando dissociação entre conhecimento e práxis( ${ }^{(8)}$. Já na década de 1990, através de uma interação, ainda tímida, entre as necessidades da realidade profissional e 0 interesse acadêmico gerado nas U niversidades, iniciou-se a geração dos resultados das inovações tecnológicas na área de E nfermagem.

$\mathrm{N}$ este sentido e diante deste rel evante panorama, nos questionamos: Com que frequência ocorre o registro de patentes na área de enfermagem? Q ue produtos/ patentes são resultantes das pesquisas em E nfermagem? Como este processo tem evoluído nas últimas duas décadas? A o considerar estas questões, este estudo teve como objetivo: analisar as patentes registradas na área de enfer magem, no período de 1990-2009, considerando que as mesmas podem configurar como um dos indicadores de seu desenvolvimento tecnológico.

\section{MET ODOLOGIA}

Trata-se de uma pesquisa do tipo documental, que "vale-se de materiais que não receberam tratamento analítico ou que ainda podem ser reelaborados de acordo com os objetivos da pesqui$\mathrm{sa}^{\prime \prime(9)}$, com abordagem qualitativa. A coleta de dados ocorreu através do acesso ao banco de registros de patentes do Instituto $\mathrm{N}$ acional de Propriedade I ndustrial (IN PI), nos meses de outubro e novembro de 2010, incluindo todos os pedidos de patentes registrados no período de 1990-2009. Como termo de busca foi utilizado o termo "enfer magem" no título e/ ou no resumo acessados na base de registro de patentes.

A pesquisa resultou em 25 registros, selecionados a partir da leitura atenta em relação à temática proposta. Estes achados foram organizados em um quadro, e a partir do mesmo, buscouse analisar: (1) a evolução tecnológica na área de enfermagem nas últimas duas décadas, (2) a autoria das patentes e (3) o tipo de utilização desta inovação tecnológica.

As informações obtidas na base de patentes do INPI foram complementadas com pesquisa pelo nome do inventor na plataforma $\mathrm{G}$ oogle e/ ou pelo seu currículo na plataforma $L$ attes do ConseIho $\mathrm{N}$ acional de D esenvolvimento Científico eT ecnológico (CNPQ) para a obtenção de dados relacionados à formação profissional/ acadêmica do mesmo.

Como se trata de pesquisa documental, Cujo conteúdo disponibilizado é de caráter público, este estudo não foi submetido ao Comitê de Ética em Pesquisa com Seres H umanos, mas todos os preceitos éticos relativos a este tipo de pesquisa foram assegurados.

\section{DISCUSSÃO E RESULTADOS}

As patentes e a evolução tecnológica na área de enfermagem

No período de 1990-2009, foram encontradas na base de patentes do I N PI 25 solicitações de registro relacionadas à área de enfermagem, que estão apresentadas no Quadro 1, a seguir.

\begin{tabular}{|c|c|c|c|}
\hline $\begin{array}{l}\text { Número do } \\
\text { Processo }\end{array}$ & $\begin{array}{l}\text { D ata do } \\
\text { Registro }\end{array}$ & T ítulo da Invenção & Inventor \\
\hline PI 0805382-0 & $02 / 12 / 2008$ & Suporte H ospitalar para pacientes acamados. & Enfermeira \\
\hline M U 8803186-1 & $01 / 12 / 2008$ & $\begin{array}{l}\text { Disposição construtiva introduzida em dispositivo } \\
\text { conector para bolsas de soluçãa parenteral. }\end{array}$ & $\begin{array}{l}\text { Engenheiro } \\
\text { de produção }\end{array}$ \\
\hline PI0806000-2 & 29/10/ 2008 & $\begin{array}{l}\text { Configuração estrutural aplicada em leito para dis- } \\
\text { posição de periféricos para informatização e informa- } \\
\text { tização de leito. }\end{array}$ & $\begin{array}{l}\text { Engenheiro } \\
\text { de produção }\end{array}$ \\
\hline M U 8702609-0 & $01 / 06 / 2007$ & Luva restritora. & Enfermeira \\
\hline PI $0701041-9$ & $13 / 04 / 2007$ & $\begin{array}{l}\text { Sistema e dispositivos de produção de aerossóis por } \\
\text { acionamento manual ou podal. }\end{array}$ & \\
\hline PI0603740-2 & $12 / 09 / 2006$ & huveiro portátil para banho de leito. & $\begin{array}{l}\text { Engenheiro } \\
\text { elétrico }\end{array}$ \\
\hline
\end{tabular}


Continuação.

\begin{tabular}{|c|c|c|c|}
\hline $\begin{array}{l}\text { Número do } \\
\text { Processo }\end{array}$ & $\begin{array}{l}\text { Data do } \\
\text { Registro }\end{array}$ & T ítulo da I nvenção & Inventor \\
\hline PI0504656-4 & $17 / 10 / 2005$ & Sonda nasoentérica deg lutível e seu método de util ização. & $\begin{array}{l}\text { Engenheiro } \\
\text { de produção }\end{array}$ \\
\hline M U 8402305-8 & $24 / 09 / 2004$ & F ixador para cânulas orotraqueais descar tável. & Enfermeira \\
\hline PI0401713-7 & $07 / 05 / 2004$ & $\begin{array}{l}\text { Papaína associada ao silicato de magnésio para cicatri- } \\
\text { zação de feridas }\end{array}$ & Enfermeira \\
\hline PI0305339-3 & $28 / 11 / 2003$ & $\begin{array}{l}\text { Processo de obtenção de cristais de 9-((1,3-dihi- } \\
\text { droxipropan-2-iloxi) metil)-2-amino-1h-purin-6(9h)- } \\
\text { ona para utilização em soro glicosado; solução a base } \\
\text { dos ditos cristais isentos de resíduos de alcalinidade; } \\
\text { processo e uso de sistema fechado no acondicionamento } \\
\text { da solução; e uso da solução e do sistema no tratamento } \\
\text { de doenças. }\end{array}$ & Bioquímico \\
\hline M U 8303466-8 & $21 / 11 / 2003$ & $\begin{array}{l}\text { Bolsa para ostomia intestinal / urinária do sistema de } \\
\text { uma peça drenável, com apêndice de } 03 \mathrm{~cm} \text { a } 04 \mathrm{~cm} \text { de } \\
\text { altura e } 05 \text { a } 06 \mathrm{~cm} \text { de comprimento. }\end{array}$ & $\begin{array}{l}\text { Engenheiro } \\
\text { de produção }\end{array}$ \\
\hline C20303075-0 & $09 / 07 / 2003$ & $\begin{array}{l}\text { Reservatório e aquecedor de água portátil com reser- } \\
\text { vatório de sabão líquido para banho de leito em pacientes } \\
\text { acamados. }\end{array}$ & $\begin{array}{l}\text { Engenheiro } \\
\text { de produção }\end{array}$ \\
\hline C10303075-0 & 09/07/ 2003 & $\begin{array}{l}\text { Reservatório e aquecedor de água portátil para banho } \\
\text { de leito em pacientes acamados em ambientes domés- } \\
\text { tico - home care. }\end{array}$ & $\begin{array}{l}\text { Engenheiro } \\
\text { de produção }\end{array}$ \\
\hline PI0303075-0 & $30 / 06 / 2003$ & $\begin{array}{l}\text { Reservatório e aquecedor de água portátil para banho } \\
\text { de leito em pacientes acamados. }\end{array}$ & $\begin{array}{l}\text { Engenheiro } \\
\text { de produção }\end{array}$ \\
\hline PI0304713-0 & $06 / 05 / 2003$ & $\begin{array}{l}\text { M étodo e sistema para a formação de interface com } \\
\text { estabelecimento de continuidade entre um sistema de } \\
\text { atendimento médico de emergência e um sistema de } \\
\text { triagem de enfermagem. }\end{array}$ & $\begin{array}{l}\text { Engenheiro } \\
\text { de produção }\end{array}$ \\
\hline PI0300521-6 & $13 / 03 / 2003$ & $\begin{array}{l}\text { Processo de obtenção de solução isotônica injetável } \\
\text { estável de gatifloxacina pré-diluída em glicose, solução } \\
\text { injetável estável de gatifloxacina pré-diluída em glicose, } \\
\text { processo de acondicionamento em sistema fechado da } \\
\text { solução injetável obtida, uso de sistema fechado para } \\
\text { acondicionamento de solução injetável de gatifloxacina, } \\
\text { uso da solução injetável a base de gatifloxacina pré- } \\
\text { diluída em glicose obtida e método de administraçãa. }\end{array}$ & Farmacêutico \\
\hline M U 8102697-8 & $08 / 11 / 2001$ & Posto móvel de saúde. & $\begin{array}{l}\text { Enfermeira e } \\
\text { Engenheiro } \\
\text { de produção }\end{array}$ \\
\hline PI9605985-0 & 13/ 12/ 1996 & $\begin{array}{l}\text { Artigo de musgo de esfagno absorvente de líquido e } \\
\text { método de manufatura do artigo absorvente. }\end{array}$ & $\begin{array}{l}\text { Engenheiro } \\
\text { de produção }\end{array}$ \\
\hline
\end{tabular}


Continuação.

\begin{tabular}{|l|l|l|l|}
\hline $\begin{array}{l}\text { Número do } \\
\text { Processo }\end{array}$ & $\begin{array}{c}\text { Data do } \\
\text { Registro }\end{array}$ & \multicolumn{1}{|c|}{ T ítulo da Invenção } & Inventor \\
\hline M U 7600670-0 & $26 / 04 / 1996$ & Disposição introduzida em lençol hospitalar. & Enfermeira \\
\hline M U 7401010-7 & $14 / 06 / 1994$ & Haste multiuso de papel, papelão ou celulosa. & $\begin{array}{l}\text { Engenheiro } \\
\text { de produção }\end{array}$ \\
\hline PI9402112-0 & $25 / 05 / 1994$ & $\begin{array}{l}\text { Suporte com alarme para recipiente com líquido de } \\
\text { infusão venosa. }\end{array}$ & $\begin{array}{l}\text { Engenheiro } \\
\text { de produção }\end{array}$ \\
\hline PI9400589-3 & $09 / 02 / 1994$ & $\begin{array}{l}\text { Suporte com alarme para recipiente com solução de uso } \\
\text { parenteral. }\end{array}$ & $\begin{array}{l}\text { Engenheiro } \\
\text { de produção }\end{array}$ \\
\hline M U 7301621-7 & $24 / 08 / 1993$ & $\begin{array}{l}\text { Dispositivo de cateterismo vascular de saída única. } \\
\text { Engenheiro } \\
\text { de produção }\end{array}$ \\
\hline M U 7301844-9 & $15 / 04 / 1993$ & Dispositivo de cateterismo vascular. & $\begin{array}{l}\text { Engenheiro } \\
\text { de produção }\end{array}$ \\
\hline M U 7201146-7 & $29 / 07 / 1992$ & Carro de higiene para pacientes acamados. & $\begin{array}{l}\text { Engenheiro } \\
\text { de produção }\end{array}$ \\
\hline
\end{tabular}

Q uadro 1 - Relação dos processos de solicitação de patentes na área de enfermagem, registrados no Banco de Patentes do IN PI, com suas respectivas datas de registro, títulos da invenção e formação acadêmica/ profissional do inventor. Brasil, 1990-2009.

Fonte: Banco de Registro de Patentes do Instituto N acional da Propriedade Industrial (I N PI), 2010.

A través da configuração disposta, verificou-se nos registros encontrados que a produção tecnológica é direcionada para a prática do cuidado, considerando a otimização do tempo e qual idade na prestação do serviço por parte da equipe de enfermagem.

Percebe-se que a produção e a ciência têm evoluído com vistas às necessidades concretas, da prática cotidiana. Contudo, observa-se que dos produtos lançados ao mercado direcionados ao cuidado de enfermagem e que contam com a capacidade de adquirir reconhecimento nos diversos ambientes de trabalho do setor de saúde, apenas $20 \%$ são desenvolvidos pelos profissionais da área. $\mathrm{A}$ liderança no desenvolvimento e registro de patentes se concentra nos profissionais da engenharias, que tem $60 \%$ das produções. 0 segmento da engenharia é o principal criador das novas e atuais tecnologias direcionadas ao mercado do cuidado, mercado este que necessita constantemente de inovações para o seu aprimoramento.

Podem-se, em princípio, levantar algumas hipóteses para explicar esta situação: 1a) a grande interação entre os grupos de pesquisa da área da engenharia com as empresas ${ }^{(3)} ; 2^{2}$ ) 0 forte financiamento que as tecnologias oriundas de natureza dura possuem, que, por excelência estão re- lacionadas às engenharias e áreas afins, favorecendo o potencial e crescente desenvolvimento de equipamentos tipo máquinas, normas e estruturas organizacionais; 3a) atualmente, as pesquisas tecnológicas no campo da enfermagem estão mais relacionadas a teorizações e processos e menos na produção de produtos; e 4aa a dificuldade do enfermeiro em patentear suas invenções.

0 número de publicações científicas sobre 0 registro de produtos na área da saúde é pequeno e limita a comparação dos resultados encontrados. $\mathrm{Na}$ área de enfermagem, foram encontrados apenas dois estudos que também discutiram sobre a quantidade de produtos patenteados. Em um estudo retrospectivo, realizado na UFSC, abrangendo o período entre 1995-2008, os pesquisadores encontraram 2467 produções técnico-científicas relacionadas aos Grupos de Pesquisa em E ducação em Enfermagem. D estas, oito foram classificadas como produtos tecnológicos, mas nenhuma foi patenteada ${ }^{(10)}$. Situação semelhante é descrita em outro estudo realizado na mesma U niversidade, no qual foram analisadas 92 dissertações de mestrado em enfermagem, apresentadas no período de 2001 a 2004, que resultaram em um grande número de tecnologias convergen- 
tes-assistenciais classificadas como de conduta, concepção, inter pretativas de situações do cliente, administrativas, de cuidado e de educação, mas nenhuma que tivesse gerado patente $\mathrm{e}^{(7)}$.

\section{As patentes produzidas em enfermagem: sua aplicabilidade e autoria frente às tendências}

Outro aspecto analisado nesta pesquisa está relacionado à utilidade das tecnologias desenvolvidas, conforme classificação apresentada na T abela 1.

T abela 1 - Classificação das Invenções de acordo com sua utilidade no serviço de Enfermagem e Cuidado. Brasil, 1990-2009.

\begin{tabular}{lcr}
\hline Utilidade & $\mathbf{n}$ & $\mathbf{\%}$ \\
\hline Higiene e Assepsia & 9 & 36 \\
Conforto do paciente312 & 3 & 12 \\
M anuseio do paciente acamado & 1 & 4 \\
Alimentação & 1 & 4 \\
Cicatrização & 1 & 4 \\
Infor matização (dados Clínicos & & \\
e procedimentos) & 2 & 8 \\
Administração de M edicação & 7 & 28 \\
Exames de Diagnóstico & 1 & 4 \\
\hline T otal & $\mathbf{2 5}$ & $\mathbf{1 0 0}$ \\
\hline
\end{tabular}

Fonte: Banco de Registro de Patentes do Instituto $\mathrm{N}$ acional da Propriedade Industrial (IN PI), 2010.

Observa-se que as necessidades básicas do usuário relacionadas à higiene e assepsia lideram (36\%) as invenções patenteadas, seguidas pelo conforto do paciente (12\%). Partindo da premissa que estas necessidades demandam de mais tempo e esforço físico do profissional, o desenvolvimento de tecnologias afins pode ser vislumbrado como algo que justifique o investimento para desenvolver e patentear inventos relacionados.

D entro da perspectiva que a tecnologia também é compreendida como estudo ou a atividade de teorias, métodos e processos científicos, para solução de problemas técnicos(1). Outro invento que se configura na mesma concepção de aprimoramento tecnológico é a administração de medicamentos (28\%). Esta atividade, em parceria com a indústria far macêutica, configura-se em um campo crescente de inovações, objetivando mais eficácia no procedimento, melhor controle de doenças e busca de maior sobrevida.

A través das informações coletadas, procurouse também fazer a relação quantitativa da distribuição dos registros de patentes real izadas no período analisado, considerando os inventores dos produtos e patentes, conforme T abela 2.

A través da comparação entre as décadas de 1990 e 2000, fica evidenciado um incremento no número de pedidos de patentes nesta última década. E sse aumento pode estar relacionado com o desenvolvimento das políticas de apoio à Ciência e T ecnologia no país e, a recriação do M inistério de Ciência e T ecnologia, em 1992, possibilitando a retomada de projetos nas diversas áreas em busca do desenvolvimento tecnológico nacional ${ }^{(11)}$.

A lém disso, a enfer magem como ciência, também cresceu na última década por meio dos incentivos direcionados a transformar idéias em inovações, em decorrência do desenvolvimento de grupos de pesquisas nos cursos de graduação e pósgraduação, gerando incremento no quantitativo de produtos patenteados por este segmento.

T abela 2 - N úmero de solicitação de registros entre 1990 e 2009 e profissão/ formação dos autor es das invenções registrados no banco de patentes. Brasil, 1990-2009.

\begin{tabular}{lrrrrrr}
\hline \multirow{2}{*}{ Profissão/ Formação } & \multicolumn{2}{c}{$\mathbf{1 9 9 0 - 1 9 9 9}$} & \multicolumn{2}{c}{$\mathbf{2 0 0 0 - 2 0 0 9}$} & \multicolumn{2}{c}{ T otal } \\
\cline { 2 - 7 } & $\mathbf{n}$ & $\mathbf{\%}$ & $\mathbf{n}$ & $\mathbf{6}$ & $\mathbf{n}$ & $\mathbf{2}$ \\
\hline Enfermeiro & 1 & 87,5 & 4 & 23 & 5 & 20 \\
Engenheiro de Produção & 7 & 12,5 & 8 & 47 & 15 & 60 \\
Farmacêutico/ Bioquímico & - & - & 2 & 12 & 2 & 8 \\
Outros profissionais & - & - & 2 & 12 & 2 & 8 \\
Co-autoria* & - & - & 1 & 6 & 1 & 4 \\
\hline Total de registros & $\mathbf{8}$ & $\mathbf{1 0 0}$ & $\mathbf{1 7}$ & $\mathbf{1 0 0}$ & $\mathbf{2 5}$ & $\mathbf{1 0 0}$ \\
\hline
\end{tabular}

Fonte: Banco de Registro de Patentes do Instituto N acional da Propriedade Industrial (IN PI), 2010.

* A invenção foi registrada por dois profissionais (um da enfermagem e outro da engenharia). 
A integração lenta, mas crescente, que vêm ocorrendo no Brasil entre a comunidade acadêmica (principalmente através dos programas de pósgraduação) e os prestadores de serviços e empresas, pode favorecer a transformação dos problemas encontrados na prática do profissional em objetos de pesquisa, e em contrapartida os resultados das mesmas, podem contribuir no aprimoramento dos serviços de saúde onde esta prática acontece ${ }^{(7)}$.

Alguns entraves têm dificultado esta integração, como por exemplo, a burocratização da academia, e o financiamento que privilegia instituições maiores e mais produtivas em detrimento das menores que não conseguem produzir mais pela carência deste financiamento. São situações que al imentam um circulo vicioso, dificul tando o desenvolvimento da própria tecnologia e, em consequência, de seu patenteamento ${ }^{(10)}$.

Independente destas e outras dificuldades, as instituições acadêmicas têm muito a contribuir em relação a esta problemática. Ela pode, por exemplo, auxiliar no aprendizado das novas tecnologias, favorecendo a aquisição das habilidades necessárias, contribuindo para o domínio da tecnologia e, consequentemente com a melhoria na segurança e organização da assistência prestada ${ }^{(6)}$.

A análise acerca das patentes na área de enfermagem também implica na reflexão sobre a situação do desenvolvimento tecnológico do país na área de saúde. No IV Encontro Nacional de PósG raduação na Ár rea de Ciências da Saúde, realizado em 2010, ao tratar da inovação em saúde na pósgraduação, buscou estabelecer caminhos para a promoção da inovação em saúde através dos cursos de pós-graduação. $\mathrm{N}$ a área de enfer magem, a proposta desenvolvida consiste em: 1ㅇ) estimular a criação de ambientes de tecnologia e inovação para desenvolvimento de modelos de cuidado de Enfermagem e Saúde, com estratégias de sustentabilidade e empreendedorismo; 2o) investir na criação de indicadores de impacto econômico e social, de tecnologia e inovação em E nfermagem e Saúde; 3) promover a criação de redes de desenvolvimento de inovação e tecnologia em E nfermagem e Saúde, para garantir um cuidado de enfermagem seguro e de excelência; 4ㅇ) propor, às agências de fomento, de Editais temáticos de tecnologia e inovação em cuidados, gerência e educação em Enfermagem e Saúde, em consonância com as políticas do SU S; 5) propor a inclusão das tecnologias de cuidado de Enfermagem na Agenda de Prio- ridades em Pesquisa e; 60) incrementar a política de expansão e articulação dos programas de PósG raduação da Área de Enfermagem, como estratégia de qualificação dos profissionais de serviço com implantação de tecnol ogias de cuidado baseado em evidências ${ }^{(12)}$.

\section{CONSIDERAÇÕES FINAIS}

$\mathrm{Na}$ área da tecnologia, pode-se observar que as produções científicas leves e leves-duras não avançaram no campo da enfermagem, e as tecnologias duras, que são inventadas, reinventadas em nossas práticas, dificilmente são registradas e, portanto reconhecidas para serem patenteadas.

Esforços devem ser estimulados para identificar pesquisas com potencial para gerar inovações, favorecendo ao pesquisador no processo de patente. Divulgar modos de realizar o procedimento de patentee, até como real izar possíveis negociações com empresas é essencial.

O setor saúde tem maior potencial para criação de processos, em detrimento daquel es que criam produtos. São procedimentos que envolvem tecnologia, socialmente importantes, pois podem ser adotados em tratamentos e na melhoria da qualidade de vida das pessoas.

No campo da pesquisa em saúde, éimportante que se avalie as inovações tecnológicas que estão em uso para que sirvam de ponto de partida para outras invenções. Os profissionais de enfermagem e os pesquisadores da área devem estabel ecer métodos de aproximação relacionados ao processo de invenção. D estaque-se que é a partir do cotidiano das unidades hospitalares ou de saúde coletiva que surgem as necessidades de melhoria materiais ou imateriais. Cabe ressaltar ainda que, tanto os profissionais da academia quanto os profissionais da prática, são geradores de produção social em saúde, mesmo que na sua inconcretude.

A tecnologia transcende a área da saúde, porque é capaz de se articular com outros setores e tecer com outras ciências um diálogo que transversaliza saberes, que é capaz de superar obstáculos, traz a habilidade para criar o novo e propiciar avanços sociais.

\section{REFERÊ NCIAS}

1 Rocha PK, Prado M L, W al M L, Carraro T E. Cuidado e tecnologia: aproximações através do M odelo de Cuidado. Rev Bras Enferm. 2008;61(1):113-6. 
2 M inistério da Saúde (BR), Secretaria Executiva. Avaliação de tecnologias em saúde: ferramentas para a gestão do SU S. Brasília (DF ); 2009.

3 Silva E M P. N ota sobre pós-graduação, desenvolvimento tecnológico e inovação [ Internet] . [ S.I.] : Professor Global; 2010 [ citado 2011 out 15]. Disponível em: http:/ / www.professorglobal.cbpf. br/ mediawiki/ index.php/ A rquivo:N otasobrePos gradT ecl nov-E vandoM irra-0.jpg.

4 Ferreira AA, G uimarães ER, Contador J C. Patente como instrumento competitivo e como fonte de informação tecnológica. G est Prod. 2009;16(2):209-21.

5 M erhy EE, Onocko R. Em busca de ferramentas analisador as das tecnologias em saúde: a informação e o dia a dia de um serviço, inter rogando e gerindo trabalho em saúde. In: M erhy EE, Onocko R, organizadores. Praxis en salud: un desafío para lo publico. São Paulo: H ucitec; 1997.

6 A rone EM , Cunha ICKO. Avaliação tecnológica como competência do enfermeiro: reflexões e pressupostos no cenário da ciência e tecnologia Rev Bras Enferm. 2006;59(4):569-72.

7 Prado M L, Backes V M S, Reibnitz KS, Cartana M HF A be $K L$, Rocha PK, et al. Produções tecnológicas em enfermagem em um curso de mestrado. Texto Contexto E nferm. 2009;18(3):475-81.
8 Paim L, M artins CR, Paese F, Bresciani HR, Callegaro GD. Demarcação histórica da enfermagem na dimensão tecnológica. T exto Contexto E nferm. 2009; 18(3):542-8.

9 Gil AC. Como elaborar projetos de pesquisa. 40a ed. São Paulo: Atlas; 2007.

10 L ino M M , Backes V M S, Canever BP, Ferraz F, Prado ML. Perfil da produção científica e tecnológica dos grupos de pesquisa em educação em enfermagem da Região Sul do Brasil. Rev L atino-A m E nfermagem [ I nternet] . 2010 [ citado 2010 nov 10] ;18 (3):165-72. D isponível em: http:/ / www.sciel o.br/ pdf/ rlae/ v18n3/ pt_22.pdf.

11 Aquino PS, M elo RP, Lopes M VO, Pinheiro AKB. Analysis of the concept of technology in nursing according to the evolutionary method. Acta Paul Enferm. 2010;23(5):690-6.

12 Relatório da Área de Enfermagem do 4ํㅡㄹ ncontro Nacional de Pós-Graduação na Área de Ciências da Saúde: como promover a inovação em saúde na pós-graduação?; 2010 nov 2-5; Bento G onçalves, Brasil [ Internet]. Porto A legre: U F CSPA; 2010 [ citado 2011 out 15]. Disponível em: http:// sis tema.ufcspa.edu.br/iv_encontro_nacional/index. php?escolha=mod/ capä/ capa.php.

\section{Endereço da autora / Dirección del autor / Author's address:}

$\mathrm{M}$ icheline Henrique A raujo da L uz Koerich

Rua Pedro Silva, 1952, Coqueiros

88080-700, Florianópolis, SC

E-mail: michelineha@ig.com.br
Recebido em: 28/ 04/ 2011

A provado em: 25/11/ 2011 